\title{
Exenatide once weekly in type 2 diabetes
}

\author{
Andre J Scheen ${ }^{*}$ \\ Division of Diabetes, Nutrition and Metabolic Disorders, Department of Medicine, CHU SartTilman, University of Liège, B-4000 Liège, \\ Belgium
}

The difficulties of maintaining long-term glycaemic control with available antidiabetic treatments in patients with type 2 diabetes have led to the need for additional treatments that can replace or be safely combined with existing agents. ${ }^{1}$ Patients with type 2 diabetes who have not achieved adequate glucose control at the maximum tolerated doses of their oral therapies have had no alternative other than insulin. ${ }^{2,3}$ However, the best insulin regimen is a matter of controversy because addition of biphasic, prandial, or basal insulin to oral therapy has been proposed. ${ }^{4}$ Furthermore, the start of insulin therapy is often delayed. ${ }^{5}$ Besides the fear of subcutaneous injections, the two main objections raised by the patient (and often also by the physician) are the risk of hypoglycaemia and weight gain.

Exenatide is the first of a new class of compounds, and has similar activity to the naturally occurring hormone glucagon-like peptide $1 .^{6}$ Administration of this drug mirrors many of the effects of glucagon-like peptide 1, improving glycaemic control through glucose-dependent stimulation of insulin secretion, suppression of glucagon secretion, slowing of gastric emptying and reduced appetite. ${ }^{7}$ Short-term ( $\leq 1$ year) phase III randomised trials showed that exenatide $10 \mu \mathrm{g}$ twice a day might enable patients with type 2 diabetes to improve glycaemic control and reduce or eliminate weight gain and the risk of hypoglycaemia. ${ }^{7}$ Similar longer term (2-3 years) effects were reported in open-label extensions from pivotal trials, which showed sustained weight reduction.

Exenatide therapy can be regarded as an alternative to insulin in patients with treatment failure on oral agents. ${ }^{7,8}$ Exenatide $10 \mu \mathrm{g}$ twice a day has been evaluated in three randomised studies, and showed similar reduction of glycated haemoglobin $A_{1 c}$ as glargine once a day or premixed biphasic insulin aspart (30/70) twice a day. ${ }^{8}$ Exenatide had the advantage of less hypoglycaemia and weight gain. Although exenatide, unlike insulin, requires no adaption of dose on the basis of glucose self-monitoring, its use does not reduce the number of daily subcutaneous injections. Adherence to diabetes drugs is vital for effective management. Several factors have been identified as challenges for taking drugs by patients with type 2 diabetes, including complexity of the regimen. ${ }^{9}$ Thus the development of a long-acting release formulation of exenatide should be regarded as major progress.

In today's Lancet, Daniel Drucker and colleagues report the DURATION-1 randomised non-inferiority trial. ${ }^{10}$ These investigators showed that long-acting exenatide $2 \mathrm{mg}$ once a week resulted in significantly greater improvements in glycaemic control than did exenatide given twice a day, with no increased risk of hypoglycaemia, lower occurrence of nausea, and similar reductions in bodyweight. ${ }^{10}$ Interestingly, such benefits were observed with long-acting exenatide when added to a broad spectrum of glucose-lowering therapies (except insulin), although today's study did not allow selection of improved responders.

However, caution is needed as with any new drug developed for the treatment of type 2 diabetes. ${ }^{11}$ Indeed, the initial enthusiasm might shift to scepticism when first concerns about effectiveness, safety, or both, arise (figure). Many glucose-lowering drugs were withdrawn from the market or their use became controversial despite early positive results. The success of any antidiabetic agent depends on efficacy for glucose control, good tolerance and safety profile, ease of use, cost (unknown for long-acting exenatide), and capacity to reduce complications. In Drucker and colleagues' study, compared with the twice-a-day exenatide regimen, the once-aweek formulation, besides obvious improved ease of use, provided the remarkable advantage of both improved efficacy on glucose control and good gastrointestinal tolerability. Currently reported adverse effects were a higher incidence of pruritus at the injection site and increased circulating antibodies.

\footnotetext{
* I am a consultant for Sanofi-Aventis, AstraZeneca, and GlaxoSmithKline, and have received lecture fees from Sanofi-Aventis.
} 
Figure: Drug development fortreatment of type 2 diabetes

Hypothetical timeline of most important oral antidiabetic drugs. Classic natural history is that confidence starts with initial enthusiasm (just before and after launch), peaks a few months (or years) after, and then generally decreases (when first disappointments occur, because of lack of response in some patients or safety concern). Last part of curve varies from drug to drug (and assumes that the drug escaped definitive withdrawal). Almost all oral antidiabetic drugs can be placed on this curve. Initial enthusiasm (as now for incretins) might shift to scepticism (and sometimes withdrawal, as with buformin, phenformin, troglitazone). As for rosiglitazone and perhaps rimonabant, major concern occurring some time after launch does not always mean demise of the drug (eg, metformin, which was almost withdrawn in the 1980s, with phenformin and buformin, is now first-choice drug for type 2 diabetes). Almost the same occurred with sulphonylureas after the UGDP trial in the $1970 \mathrm{~s}$.

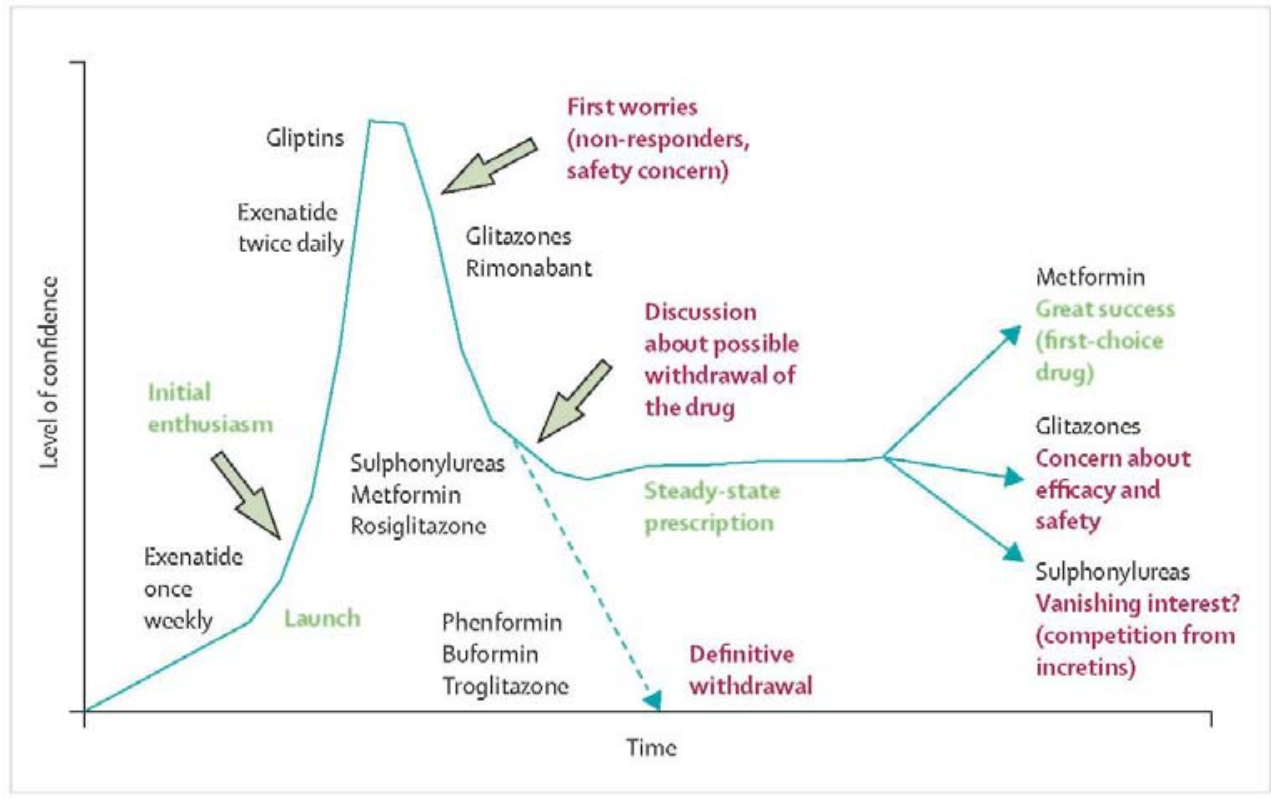

The durability of treatment effects on surrogate variables shown after 30 weeks on long-acting exenatide, as in DURATION-1, should be further assessed in longer-term trials. Head-to-head studies that compare exenatide once a week with liraglutide, ${ }^{12}$ a glucagon-like peptide 1 analogue that has a longer half-life and better dosing regimen (once a day) than the classic formulation of exenatide twice a day, would also be useful and interesting. Additional studies should better evaluate patient-oriented factors that might favour exenatide over insulin use. ${ }^{8,13,14}$ Last, positive effects of exenatide on microangiopathy complications or cardiovascular events have yet to be seen, ${ }^{7,14}$ the ultimate goal in diabetes management.

Exenatide is not yet considered in international consensus guidelines. ${ }^{2,3}$ The updated NICE guideline recommended to offer exenatide only when insulin would otherwise be started, obesity is a specific problem (body-mass index $>35 \mathrm{~kg} / \mathrm{m}^{2}$ ), and the need for a high dose of insulin is likely. ${ }^{15}$ When the once-a-week exenatide formulation becomes available, after confirmation and extension of today's positive results, this new strategy might substantially change the management of type 2 diabetes. Extraordinarily, a once-a-week drug administration could favourably and durably affect the control of basal and postprandial glucose, which results from an exquisite continuous timely feedback loop.

\section{References}

1 Stumvoll M, Goldstein BJ, van Haeften TW. Type 2 diabetes: principles of pathogenesis and therapy. Lancet 2005; 365:1333-46.

2 Nathan DM, BuseJB, Davidson MB, et al. Management of hyperglycaemia in type 2 diabetes: a consensus algorithm for the initiation and adjustment of therapy. A consensus statement from the American Diabetes Association and the European Association for the Study of Diabetes. Diabetologia 2006; 49:1711-21.

3 Nathan DM, BuseJB, Davidson MB, et al. Management of hyperglycaemia in type 2 diabetes: a consensus algorithm for the initiation and adjustment of therapy. A consensus statement from the American Diabetes Association and the European Association for the Study of Diabetes. Update regarding the thiazolidinediones. Diabetologia 2008; 51: 8-11.

4 Holman RR, Thorne Kl, Farmer AJ, et al,for the 4-T Study Group. Addition of biphasic, prandial, or basal insulin to oral therapy in type 2 diabetes. $N$ Engl J Med 2007; 357:1716-30.

5 Peyrot M, Rubin RR, Lauritzen T, et al, on behalf of the International DAWN Advisory Panel. Resistance to insulin therapy among patients and providers: results of the cross-national Diabetes Attitudes, Wishes, and Needs (DAWN) study. Diabetes Care 2005; 28: 267379

6 Drucker DJ, Nauck MA. The incretin system: glucagon-like peptide-1 receptor agonists and dipeptidyl peptidase-4 inhibitors in type 2 
Published in: Lancet (2008), vol. 372, pp. 1197-1198.

Status: Postprint (Author's version)

diabetes. Lancet 2006; 368:1696-705.

7 Cvetkovic RS, Plosker GL. Exenatide: a review of its use in patients with type 2 diabetes mellitus (as an adjunctto metformin and/or a sulfonylurea). Drugs 2007; 67: 935-54.

8 Van Gaal LF, Gutkin SW, Nauck MA. Exploiting the antidiabetic properties of incretins to treat type 2 diabetes mellitus: glucagon-like peptide-1 receptor agonists or insulin for patients with inadequate glycemic control? Eur J Endocrinol 2008; 158: 773-84.

9 Odegard PS, Capoccia K. Medication taking and diabetes: a systematic review of the literature. Diabetes Educ 2007; 33:1014-29.

10 Drucker DJ, BuseJB, Taylor K, et al, for the DURATION-1 Study Group. Exenatide once weekly versus twice dai ly for the treatment of type 2 diabetes: a randomised, open-label, non-inferiority study. Lancet 2008; published online Sept 8. DOI:10.1016/S0140$6736(08) 61206-4$

11 Nathan DM. Finding new treatments for diabetes: how many, how fast and how good? N EnglJ Med 2007; 365: 437-40.

12 Visbøll T. Liraglutide: a once-daily GLP-1 analogue for the treatment for the treatment of type 2 diabetes mellitus. Expert Opin Investig Drugs 2007; 16: 231-37

13 Edwards KL, Alvarez C, Irons BK, Fields J. Third-line selection for patients with type 2 diabetes mellitus uncontrolled with sulfonylureas and metformin. Pharmacotherapy 2008; 28: 506-21.

14 Guerci B, Salaun Martin C. Exenatide. Its position in the treatment of type 2 diabetes. Ann Endocrinol 2008; 69: 201-09.

15 Home Ph, Mant J, Diaz J, Turner C, on behalf of the Guideline Development Group. Management of type 2 diabetes: summary of updated NICE guidance. BMJ 2008; 336:1306-08. 